University of Nebraska - Lincoln

DigitalCommons@University of Nebraska - Lincoln

\title{
Same-Destination-Intermediate Grouping vs. End-to-End Grouping for Waveband Switching in WDM Mesh Networks
}

\author{
Mengke Li \\ Wang Yao \\ Byrav Ramamurthy \\ University of Nebraska-Lincoln, bramamurthy2@unl.edu
}

Follow this and additional works at: https://digitalcommons.unl.edu/cseconfwork

Part of the Computer Sciences Commons

Li, Mengke; Yao, Wang; and Ramamurthy, Byrav, "Same-Destination-Intermediate Grouping vs. End-to-End Grouping for Waveband Switching in WDM Mesh Networks" (2005). CSE Conference and Workshop Papers. 74.

https://digitalcommons.unl.edu/cseconfwork/74

This Article is brought to you for free and open access by the Computer Science and Engineering, Department of at DigitalCommons@University of Nebraska - Lincoln. It has been accepted for inclusion in CSE Conference and Workshop Papers by an authorized administrator of DigitalCommons@University of Nebraska - Lincoln. 


\title{
Same-Destination-Intermediate Grouping vs. End-to-End Grouping for Waveband Switching in WDM Mesh Networks
}

\author{
Mengke Li, Wang Yao, and Byrav Ramamurthy \\ Department of Computer Science and Engineering \\ University of Nebraska-Lincoln, USA \\ Email: \{mli, wyao,byrav\}@cse.unl.edu
}

\begin{abstract}
We investigate waveband switching (WBS) with different grouping strategies in wavelength-division multiplexing (WDM) mesh networks. End-to-end waveband switching (ETEWBS) and same-destination-intermediate waveband switching (SD-IT-WBS) are analyzed and compared in terms of blocking probability and cost savings. First, an analytical model for ETEWBS is proposed to determine the network blocking probability in a mesh network. For SD-IT-WBS, a simple waveband switching algorithm is presented. An analytical model to determine the network blocking probability is proposed for SD-IT-WBS based on the algorithm. The analytical results are validated by comparing with simulation results. Both results match well and show that ETE-WBS slightly outperforms SD-IT-WBS in terms of blocking probability. On the other hand, simulation results show that SD-IT-WBS outperforms ETE-WBS in terms of cost savings.
\end{abstract}

\section{INTRODUCTION}

Wavelength-division multiplexing (WDM) is the most viable technique for bandwidth increase in wide-area backbone networks. In WDM networks, the fiber bandwidth has been divided into a set of wavelengths and a waveband is a specific set of wavelengths. The waveband switched along a route as a single bundle forms a waveband-route. In this way, a wavebandroute can reduce the number of ports used compared with the corresponding wavelength-routes (lightpaths) as discussed in [1], [2], and [3]. Thus, adopting the waveband switching (WBS) technique can generate more revenue by minimizing the switching and transmission costs in the optical domain of WDM networks. However, adopting the WBS technique adds more constraints on provisioning a call, such as the waveband availability and the grouping efficiency of wavelengths into wavebands. These additional constraints affect the network performance in terms of blocking probability. Thus, it is important to analyze the network blocking probability of a WDM network with WBS functionality.

There are different schemes to provision a call along a waveband-route, which result in different network blocking probabilities and network throughputs. While previous studies on WBS focus on achieving maximum cost savings, the blocking probability analysis and comparison of different WBS schemes remain unexplored. This paper compares two

This work was supported in part by the U. S. National Science Foundation grants (ANI-0074121 and EPS-0091900) and the UNL Program of Excellence PRISM Priority Initiative.
WBS schemes, end-to-end waveband switching (ETE-WBS) and same-destination-intermediate waveband switching (SDIT-WBS). ETE-WBS only groups wavelengths for calls with the same source-destination pair into a waveband, as shown in [1]-[4]. Thus, a waveband-route for a call is generated from its source node and terminated at its destination node. SD-ITWBS groups wavelengths for calls with the same destination into a waveband at an intermediate node. Thus, a wavebandroute is generated at the intermediate node, transmitted along the following common links, and terminated at the destination node. Note that another form of intermediate grouping could group arbitrary set of wavelengths into a waveband along a common set of links. We do not consider this case.

The advantage of SD-IT-WBS over ETE-WBS in port savings is straight-forward. However, it cannot be inferred, in general, that SD-IT-WBS can achieve better performance in terms of network blocking probability. Thus, it is important to study and compare the performance of these two WBS schemes. To the best of our knowledge, no previous study has ever addressed this topic. We analyze the blocking probability of ETE-WBS and SD-IT-WBS based on the generalized reduced load approximation presented in [5] [6] [7], which is also known as the Erlang fixed-point approximation. We first propose an analytical model for ETE-WBS to evaluate the network blocking probability across a mesh network. The analytical model is based on fixed routing and random wavelength/waveband assignment. For SD-IT-WBS, a simple algorithm is presented based on fixed routing, random wavelength/waveband assignment, and random selection of a candidate waveband-route. An analytical model is then proposed for SD-IT-WBS based on the algorithm.

\section{Network And Traffic Model Assumptions}

The network consists of $N$ nodes and $J$ uni-directional links. Each node is a multi-granular optical crossconnect (MGOXC) capable of routing calls at different granularities. Each link is a fiber link carrying $W$ wavelengths and $B$ wavebands. Each waveband may consist of at most $G$ specific wavelengths. A fiber link may contain both wavelengths and wavebands. For each call, there is a fixed route determined by the shortest path routing algorithm. Traffic for a node pair $c_{i}(s, d)$ arrives according to a Poisson distribution with a rate $\lambda_{i}$ requiring a full wavelength, where $s$ is its source node and $d$ is its 
destination node. The set of calls in the network is denoted as $C$, where $C=\left\{c_{1}, c_{2}, \ldots, c_{K}\right\}$ and $K=(N-1) * N$. Each call holding time is exponentially distributed with a unit mean $\mu_{i}=1$. The mean offered load of the node pair $c_{i}(s, d)$ is $\rho_{i}=$ $\frac{\lambda_{i}}{\mu_{i}}=\lambda_{i}$. The fixed route for the call $c_{i}(s, d)$ is denoted as $L_{c_{i}}$. $\mathrm{A}$ call along a wavelength/waveband-route is constrained by wavelength/waveband availability and wavelength/waveband continuity separately. A waveband $b_{i}$ is constrained to wavelengths $i, i+B, \ldots, i+(G-1) B$. The waveband/wavelength assigned to a waveband/wavelength-route is chosen randomly. We assume that the free wavelength/waveband distribution is independent from link to link.

To be cost efficient, a waveband should consist of at least two wavelengths. Thus, in ETE-WBS, if there are at least 2 calls with the same source-destination pair, a random free waveband is assigned along the fixed route. Wavelengths are assigned randomly to the calls according to the above specification. Otherwise, no waveband-route is set up. Wavelengthroutes are set up along the fixed routes assigned with random free wavelengths. In SD-IT-WBS, if there are at least 2 calls with some common links along their fixed routes and they are destined to the same destination, a waveband-route is set up along the common links and a free waveband is assigned randomly. Wavelengths are assigned to the calls randomly according to the the above specification. Otherwise, the calls are provisioned through wavelength-routes.

\section{A. Traffic Model}

The network blocking probability is the ratio of the total blocked load versus the total offered load. The total blocked load of a network depends on the availability of the links. The availability of a link is influenced by the traffic arrival rate on that link, which relies on the arrival rate of end-to-end calls that use this link in their routes. Because of the possible blocking of a call, the offered load on a link is reduced. Thus, the call arrival rate of a link in turn depends on the network blocking probability.

Let $A(l)$ denote the offered load on route $l$. Let $A(j)$ be the offered load on link $j$. Let $A(j, l)$ be the offered load on link $j$ due to the calls on the route $l$. Let $p_{w}(l)$ be the probability that the route $l$ is a wavelength-route and $B_{w}(l)$ be the blocking probability of a call on the wavelength-route $l$. Let $p_{b}(l)$ be the probability that the route $l$ is a waveband-route and $B_{b}(l)$ be the blocking probability of a call on the waveband-route $l$. Considering the blocking on the route $l$, if the link $j$ is on the route $l$, we have

$$
A(j, l)=A(l) \times\left[1-p_{w}(l) \times B_{w}(l)-p_{b}(l) \times B_{b}(l)\right] .
$$

Otherwise, $A(j, l)=0$. The offered load on the link $j$ is the sum of all the calls that include the link $j$ as a hop. Thus, the offered traffic on the link $j$ is

$$
A(j)=\sum_{\forall l: j \in l} A(j, l) .
$$

Note that the traffic on a link is the superposition of the overflow traffic from different calls. The overflow traffic from a call is no longer Poisson because of the blocking events. Actually it is bursty with a variance greater than its mean. The superposition of bursty traffic is bursty. Thus, the traffic on a link is not Poisson. However, to facilitate the analysis, the link traffic is approximated as Poisson. This approximation is reasonable for a low loss network and is a standard approximation in circuit-switched networks.

\section{END-TO-END WAVEBAND SWITCHING BLOCKING PROBABILITY ANALYSIS}

In ETE-WBS, a call can be provisioned if there are enough free resources. A call is blocked if there is no common free wavelength along the route. If the call satisfies the waveband grouping requirements for ETE-WBS, the call is also blocked if there is no common free waveband along the route. The blocking probability of a link is derived first, based on which the blocking probability of a wavelength-route is derived. Then the probability that a call is provisioned in a waveband-route is analyzed. After that, the blocking probability of a wavebandroute is analyzed. The whole network blocking probability is derived finally.

\section{A. Blocking Probability on a Link}

To calculate the blocking probability on a link, the link is modelled as a Markov process. Assume that the offered traffic of a link $j$ is Poisson with a rate $\lambda_{j}$. The state of the link is the number of free wavelengths on it, denoted as $\pi_{j}$. The holding time of each call is exponentially distributed with a unit mean. Consequently, the arriving and serving behaviors on the link $j$ form an $M / M / W / W$ Markov system. Let $p\left(\pi_{j}=k\right)$ be the probability that the link $j$ has $k$ free wavelengths. By solving the Markov chain, the free wavelength distribution is

$$
p\left(\pi_{j}=k\right)=\frac{\prod_{i=1}^{k}(W-i+1)}{\left(\lambda_{j}\right)^{k}} p\left(\pi_{j}=0\right),
$$

where

$$
p\left(\pi_{j}=0\right)=\left[1+\sum_{i=1}^{W} \frac{\prod_{x=1}^{i}(W-x+1)}{\left(\lambda_{j}\right)^{i}}\right]^{-1} .
$$

The blocking probability on link $j$ is given by (4).

\section{B. Blocking Probability on a Wavelength-Route}

The blocking probability on a wavelength-route depends on the free wavelength distribution of the links along the route. It can be derived recursively similar to [7]. We start from the calculation of the blocking probability for a two-hop wavelength-route $L_{c}$ for a call $c(s, d)$. Assume wavelengthroute $L_{c}^{2}$ is a two-hop route consisting of links $j_{1}$ and $j_{2}$. The probability that the wavelength-route $L_{c}^{2}$ has $k$ common free wavelengths given that the link $j_{1}$ has $k_{1}$ free wavelengths and the link $j_{2}$ has $k_{2}$ free wavelengths is

$$
p\left(L_{c}^{2}=k \mid L_{j_{1}}^{1}=k_{1}, L_{j_{2}}^{1}=k_{2}\right)=\frac{\left(\begin{array}{c}
k_{1} \\
k
\end{array}\right)\left(\begin{array}{c}
W-k_{1} \\
k_{2}-k
\end{array}\right)}{\left(\begin{array}{l}
W \\
k_{2}
\end{array}\right)},
$$

when $\max \left(0, k_{1}+k_{2}-W\right) \leq k \leq \min \left(k_{1}, k_{2}\right)$. Otherwise, $p\left(L_{c}^{2}=k \mid L_{j_{1}}^{1}=k_{1}, L_{j_{2}}^{1}=\overline{k_{2}}\right)=0$. The links occupancies 
along the route are independent from each other. Thus, the probability that there are $k$ common free wavelengths in the two-hop wavelength-route $L_{c}^{2}$ is:

$$
\begin{aligned}
p\left(L_{c}^{2}=k\right) & =\sum_{i=0}^{W} \sum_{j=0}^{W}\left[p\left(L_{c}^{2}=k \mid L_{k_{1}}^{1}=i, L_{k_{2}}^{1}=j\right)\right. \\
& \left.\times p\left(L_{k_{1}}^{1}=i\right) \times p\left(L_{k_{2}}^{1}=j\right)\right]
\end{aligned}
$$

where $p\left(L_{k_{1}}^{1}=i\right)$ and $p\left(L_{k_{2}}^{1}=j\right)$ can be calculated by using (3). For the $h$-hop wavelength-route $L_{c}^{h}$, where $h>2$, the probability that there are $k$ common free wavelengths can be derived recursively.

$$
\begin{aligned}
p\left(L_{c}^{h}=k\right) & =\sum_{i=0}^{W} \sum_{j=0}^{W}\left[p\left(L_{c}^{h}=k \mid L_{y}^{h-1}=i, L_{k_{h}}^{1}=j\right)\right. \\
& \left.\times p\left(L_{y}^{h-1}=i\right) \times p\left(L_{k_{h}}^{1}=j\right)\right] .
\end{aligned}
$$

The blocking probability of the wavelength-route $L_{c}^{h}$ is $p\left(L_{c}^{h}=0\right)$.

\section{Probability of a Call Being Carried in a Waveband-Route}

ETE-WBS tries to set up a waveband-route if there are at least 2 calls with the same source-destination pair. Otherwise, the arrival calls are provisioned along wavelength-routes. The call arrival rate for a node pair $c(s, d)$ along route $L_{c}$ is Poisson with a rate $\lambda_{c(s, d)}$. Because the waveband is randomly chosen from the free wavebands and there are at most $B$ wavebands along the route. The call arrival rate for a waveband-route $B_{c}$ is Poisson with a rate $\lambda_{B_{c}}=\frac{\lambda_{c(s, d)}}{B}$. The holding time for calls on a waveband-route is exponentially distributed with a unit mean. The waveband-route can accommodate at most $G$ calls. Thus, the holding time for a waveband-route is exponential with a unit mean. Consequently, the arrival and serving behaviors on a waveband-route form an $\mathrm{M} / \mathrm{M} / \mathrm{G} / \mathrm{G}$ Markov system. The state of the waveband-route is the number of calls on the route.

Let $p\left(\pi_{B_{c}}=k\right)$ be the probability that the wavebandroute $B_{c}$ has $k$ calls. By solving the Markov chain, the call distribution on the waveband-route can be computed. For $k>0, p\left(\pi_{B_{c}}=k\right)$ is similar to (3) except that the queuing capacity is $G$ instead of $W$ and the traffic rate is $\lambda_{B_{c}}$ instead of $\lambda_{j}$. For $k=0, p\left(\pi_{B_{c}}=0\right)$ can be expressed in the same manner as (4) except for the difference in queuing capacity and traffic rate. If the waveband-route $B_{c}$ does not have more than one call, the waveband grouping requirement is not satisfied. Thus, the call should not be provisioned along the wavebandroute $B_{c}$. Let $p_{w}^{c}$ be the probability that the call $c(s, d)$ is not in a waveband-route, then

$$
p_{w}^{c}=p\left(\pi_{B_{c}}=0\right)+p\left(\pi_{B_{c}}=1\right) .
$$

Let $p_{b}^{c}$ be the probability that the call $c(s, d)$ is in a wavebandroute, then

$$
p_{b}^{c}=1-p_{w}^{c} .
$$

According to the results in [1] [2] [3], a waveband-route with less than 2 hops does not gain any advantage in cost savings.
Thus, a waveband-route should have at least 2 hops. No call with a shorter fixed-route can be grouped in a waveband-route. Thus, if $h\left(L_{c}\right)<2, p_{w}^{c}=1$.

\section{Blocking Probability on a Waveband-Route}

The blocking probability on a waveband-route depends on the free waveband distribution of the links along the route. The offered load on link $j$ is Poisson with a rate $\lambda_{j}$. There are at most $G$ wavelengths in a waveband. Thus, the offered load at the waveband level on the link $j$ is Poisson with a rate $\lambda_{b j}=\frac{\lambda_{j}}{G}$. For a call in the waveband-route, a free wavelength is assigned randomly among the specific wavelengths. The holding time of each call is exponentially distributed with a unit mean. Thus, the holding time for calls at the waveband level on the link $j$ is exponentially distributed with a unit mean. Consequently, the arrival and the serving behaviors at the waveband level on the link $j$ form an $\mathrm{M} / \mathrm{M} / \mathrm{B} / \mathrm{B}$ Markov system. The state of the Markov process is the number of free wavebands on the link $j$, which can accommodate at most $B$ wavebands.

By solving the Markov chain, the free waveband distribution on the link $j$ can be computed. Let $p\left(\pi_{j}^{b}=k\right)$ be the probability that there are $k$ free wavebands on the link $j$. For $k>0, p\left(\pi_{j}^{b}=k\right)$ is similar to (3) except that the queuing capacity is $B$ instead of $W$ and the traffic rate is $\lambda_{b j}$ instead of $\lambda_{j}$. For $k=0, p\left(\pi_{j}^{b}=0\right)$ can be expressed in the same manner as (4) except for the difference in queuing capacity and traffic rate.

A waveband-route is constrained by its waveband continuity. To calculate the blocking probability of a wavebandroute, the same recursive method described in Section III-B for an $h$-hop wavelength-route is used. The expression of free waveband distribution $p\left(B_{c}^{h}=k\right)$ along an $h$-hop wavebandroute $B_{c}$ is similar to (7). The blocking probability of the $h$-hop waveband-route $B_{c}$ is $p\left(B_{c}^{h}=0\right)$.

\section{E. Network Blocking Probability}

The blocking probability of a call depends on the blocking probability of its wavelength-route, the blocking probability of its waveband-route, and the probability that the call is in the waveband-route. Let $B_{c}\left(s, d, L_{c}\right)$ be the blocking probability of call $c(s, d)$ on route $L_{c}$, then

$$
B_{c}\left(s, d, L_{c}\right)=p\left(L_{c}^{h}=0\right) \times p_{w}^{c}+p\left(B_{c}^{h}=0\right) \times p_{b}^{c} .
$$

The network blocking probability is the ratio of the total blocked load versus the total offered load, which is

$$
B=\frac{\sum_{c \in C} B_{c}\left(s, d, L_{c}\right)}{\sum_{c \in C} c} .
$$

To compute the network blocking probability, we need to solve the set of nonlinear fixed point equations. By successively applying (2), (7), (8), (9), (10), (11), and the equations about waveband-route blocking probability, a convergence point can be found as the solution. 


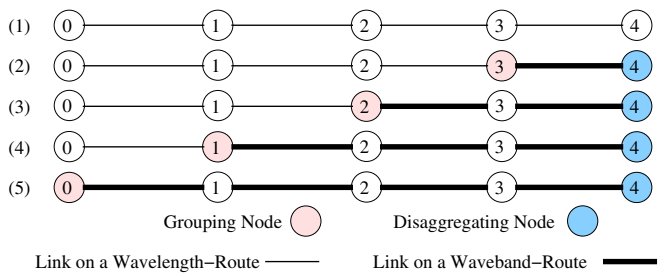

Fig. 1. An example of selecting a candidate waveband-route for a call along a 4-hop path, where case (2) should be eliminated considering the wavebandroute length constraint.

\section{Same-Destination-Intermediate WaVEband SWITCHING ALGORITHM}

In SD-IT-WBS, a call can traverse a waveband-route, which should be destined to the same destination. Generally, a call with an $h$-hop fixed route, where $h \geq 2$, can traverse $h$ different waveband-routes as illustrated in Fig. 1. As a 1-hop waveband-route does not contribute to cost savings, it should be eliminated. Thus, there are $h-1$ candidate waveband-routes. Given that a call traverses along a wavebandroute, there is a grouping node and a disaggregating node. The waveband-route is generated at the grouping node and terminated at the disaggregating node, which is the destination node of the call. By selecting different candidate wavebandroutes, the call can be provisioned with different blocking probabilities. Different strategies in selecting the candidate waveband-route result in different SD-IT-WBS algorithms.

This paper considers the random selection of a candidate waveband-route to facilitate analysis. Other approaches, such as sequential searching and exhaustive searching, can lead to smaller blocking probabilities. However, they are very difficult to be accurately analyzed and the computations are time consuming. The corresponding SD-IT-WBS algorithm is called the random SD-IT-WBS algorithm. It first randomly selects a candidate waveband-route. The algorithm then checks the availability of the selected route. If the call cannot be provisioned, the algorithm tries to provisioning it using a wavelength-route. The detailed description of the random SDIT-WBS algorithm is shown in Fig. 2. The inputs of the algorithm are the network topology, the call, the fixed-route for the call, and the utilization of wavelengths/wavebands along the route.

\section{SAme-Destination-Intermediate Waveband SWitching Blocking Probability ANALysis}

This section analyzes the blocking probability of the random SD-IT-WBS algorithm. Some of the analytical results from Section III can be used, which are the traffic model, the blocking probability of a wavelength-route, the blocking probability of a waveband-route, and the probability of a call $c\left(s, d, L_{c}\right)$ in a waveband-route if $h\left(L_{c}\right) \leq 2$, where $h\left(L_{c}\right)$ is the hop count of its fixed route $L_{c}$. The network model is the same except that the call can be grouped into a wavebandroute at an intermediate node. The disaggregating node for both SD-IT-WBS and ETE-WBS is the destination node of the call. The differences between SD-IT-WBS and ETE-WBS are

\section{Random SD-IT-WBS Algorithm}

Input: The network topology and a call $c\left(s, d, L_{c}\right)$, where $s$ is its source node, $d$ is its destination node, and $L_{c}$ is its fixed-route. Output: An intermediate waveband-route $B_{c}=\left\{L_{c}, r, b, w\right\}$ or a wavelength-route $W_{c}=\left\{L_{c}, w\right\}$.

1). Randomly select a candidate waveband-route $r$ along $L_{c}$, where $h(r) \geq 2$. If no such route, go to step 4). Otherwise, continue.

2). Search for a satisfied active waveband $b$ on route $r$ with a satisfied random free wavelength $w$. If succeeded, continue. Otherwise, go to Step 4).

3). Check whether $w$ is free on the remaining route $L_{c}-r$. If failed, go to step 4). Otherwise, return the intermediate waveband-route $B_{c}=\left\{L_{c}, r, b, w\right\}$.

4). Search for a free wavelength $w$ on route $L_{c}$. If failed, return NULL. Otherwise, return the wavelength-route $W_{c}=$ $\left\{L_{c}, w\right\}$.

Fig. 2. The random same-destination-intermediate waveband switching algorithm (SD-IT-WBS) with random selection of a candidate wavebandroute, where $h(r)$ is the hop count of route r.

the probability of the call being carried in a waveband-route and the blocking probability of the call when $h\left(L_{c}\right)>2$.

\section{A. Probability of a Call Being Carried in a Waveband-Route}

If a call has a fixed-route $L_{c}$ and $h\left(L_{c}\right)<2$, the probability that it is in a waveband-route is $p_{c}^{b}=0$. If $h\left(L_{c}\right)=2, p_{c}^{b}$ is the same as discussed in Section III-C. If $h\left(L_{c}\right)>2$, the probability is analyzed as follows. As shown in Fig. 1, a call along an $h$-hop route $L_{c}^{h}$ can be provisioned in one of the $h-1$ candidate waveband-routes or the wavelength-route. Each $h-1$ candidate waveband-route has the same probability to be selected. Let $\hat{B}_{c}$ be the set of candidate waveband-routes for call $c$. Let $B_{c}^{i}$ be the candidate waveband-routes with $i$ hops. Then, $\hat{B}_{c}=\left\{B_{c}^{i} \mid i=2, \ldots, h\right\}$ considering the wavebandroute length constraint. The probability of choosing one of them is $\frac{1}{h-1}$. Let $B_{c}^{i}$ be a candidate waveband-route for the call. Let $\hat{L_{c}^{i}}=\left\{L_{c^{\prime}}^{h^{\prime}} \mid c^{\prime} \in C\right\}$ be the set of fixed routes for calls that include $B_{c}^{i}$ as one of their candidate wavebandroutes. Correspondingly, the set of probabilities for these calls selecting candidate waveband-route $B_{c}^{i}$ is $\left\{p_{L_{c^{\prime}}^{h^{\prime}}}\left(B_{c}^{i}\right)=\right.$ $\left.\frac{1}{h^{\prime}-1} \mid c^{\prime} \in C\right\}$. Let $P_{B_{c}^{i}}(\pi=0)$ be the probability that no call in $\hat{L_{c}^{i}}$ selects the candidate waveband-route $B_{c}^{i}$, then

$$
P_{B_{c}^{i}}(\pi=0)=\prod_{c_{i} \in \hat{L}_{c}^{i}} \frac{h\left(c_{i}\right)-2}{h\left(c_{i}\right)-1},
$$

where $h\left(c_{i}\right)$ is the number of hops to route call $c_{i}$. Let $P_{B_{c}^{i}}(\pi=1)$ be the probability that only one call in $\hat{L_{c}^{i}}$ selects $B_{c}^{i}$, then

$P_{B_{c}^{i}}(\pi=1)=\sum_{c_{j} \in \hat{L}_{c}^{i}}\left\{\frac{1}{h\left(c_{j}\right)-1} \times \prod_{c_{i} \in \hat{L}_{c}^{i}-c_{j}}\left[\frac{h\left(c_{i}\right)-2}{h\left(c_{i}\right)-1} \times \frac{1}{\left|\hat{L_{c}^{i}}\right|}\right]\right\}$

Because a waveband-route must contain more than one call to be cost efficient, the probability that the call is not in the waveband-route $B_{c}^{i}$ is

$$
P_{\bar{B}_{c}^{i}}^{c}=P_{B_{c}^{i}}(\pi=0)+P_{B_{c}^{i}}(\pi=1) .
$$


The probability of the call being carried along $B_{c}^{i}$ is

$$
P_{B_{c}^{i}}=1-P_{B_{c}^{i}}^{c} \text {. }
$$

Thus, the probability that the call is not in any of the candidate waveband-routes is

$$
P_{w}^{c}=\sum_{B_{c}^{i} \in B_{c}}\left[P_{\bar{B}_{c}^{i}}^{c} \times \frac{1}{h-1}\right] .
$$

\section{B. Network Blocking Probability}

The blocking probability of a call $c$ can be calculated by using the same method described in Section III if $h\left(L_{c}\right) \leq 2$. If $h\left(L_{c}\right)>2$, the blocking probability of a call is analyzed as follows.

A call $c(s, d)$ is blocked if it is blocked on the selected candidate waveband-route or along the remaining wavelengthlinks. Let $W_{c}^{h-i}$ be the remaining wavelength-links besides the waveband-route $B_{c}^{i}$ along the fixed route $L_{c}$. Let $p\left(W_{c}^{h-i}=0\right)$ be the probability that there is no common free wavelength along the links. The probability $p\left(W_{c}^{h-i}=0\right)$ can be deduced according to (7). The probability that the call $c$ is blocked along waveband-route $B_{c}^{i}$ is $p\left(B_{c}^{i}=0\right)$, which can be calculated as shown in Section III-D. Because of the link independence assumption, the blocking probability of the call along the candidate intermediate waveband-route $B_{c}^{i}$ is

$$
p_{b}\left(B_{c}^{i}=0\right)=p\left(W_{c}^{h-i}=0\right)+p\left(B_{c}^{i}=0\right) .
$$

The blocking probability of the call along the wavelengthroute is $p\left(L_{c}^{h}=0\right)$, where $h$ is the hop count of $L_{c}$. This probability can also be calculated according to (7). Thus, the blocking probability of the call $c$ is

$$
\begin{aligned}
B_{c}\left(s, d, L_{c}\right) & =p\left(L_{c}^{h}=0\right) \times P_{w}^{c}+ \\
& \sum_{B_{c}^{i} \in \hat{B}_{c}^{h}}\left[p_{b}\left(B_{c}^{i}=0\right) \times P_{B_{c}^{i}} \times \frac{1}{h\left(L_{c}\right)-1}\right],
\end{aligned}
$$

if $h\left(L_{c}\right)>2$.

The network blocking probability is the ratio of the total blocked load versus the total offered load. Let B be the network blocking probability, then

$$
B=\frac{\sum_{c \in C} B_{c}\left(s, d, L_{c}\right)}{\sum_{c \in C} c} .
$$

The network blocking probability is the convergence point of the set of nonlinear fixed point equations. By successively applying (2), (7), (8), (9), (10), (11), (16), (18), (19), and the equations about waveband-route blocking probability, the convergence point can be deduced as the solution.

\section{Numerical Results}

This section compares the performance of ETE-WBS and SD-IT-WBS on the NSF network with 14 nodes and 21 bidirectional links in terms of network blocking probability and cost savings. The cost savings are presented by the port saving ratios because the network provisioning cost is dominated by the MG-OXC ports used. The port saving ratio as defined in [3] is the ratio of the total number of ports saved through WBS versus the total number of ports used

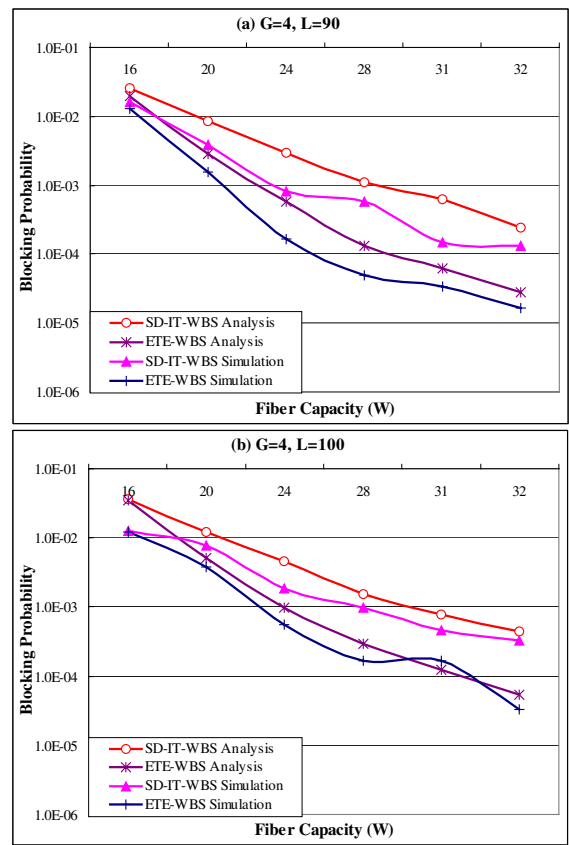

Fig. 3. Comparison of SD-IT-WBS and ETE-WBS in terms of blocking probability. (a) The NSF network, $\mathrm{W}=16, \mathrm{G}=4, \mathrm{~L}=90$, (b) The NSF network, $\mathrm{W}=16, \mathrm{G}=4, \mathrm{~L}=100$

through wavelength routing only in a WDM network. Note that since the cost of an $\mathrm{O} / \mathrm{O} / \mathrm{O}$ port is about $\frac{1}{5}$ of an $\mathrm{O} / \mathrm{E} / \mathrm{O}$ 's, the real cost saving ratio might be higher than the port saving ratio presented in this section. In addition, the accuracy of the analytical models are validated by comparing them with the simulation results.

Each simulation result is obtained by running $1,000,000$ calls. The Poisson traffic is generated for the network with a rate $\lambda$. The traffic is uniformly distributed among the set of calls. The traffic arrival rate of each node pair in the network can be derived accordingly. The call holding time is exponential with a unit mean time $\mu=1$. The network load in terms of Erlang is $\rho=\frac{\lambda}{\mu}$. The following notations are used in this section. $W$ is the maximum number of wavelengths on a fiber link. $G$ is the maximum number of active (used) wavelengths in an active (used) waveband. $B$ is the maximum number of active (used) wavebands on a fiber link. $L$ is the network load in terms of Erlang.

\section{A. Comparisons of the Waveband Switching Schemes}

Fig. 3 shows the blocking probabilities of the two WBS schemes, SD-IT-WBS and ETE-WBS, on the NSF network with $w=16$. As can be seen, the blocking probability changes with the fiber capacity for both schemes. Also, the network blocking probability decreases as the fiber capacity increases for both schemes. This trend has been proved and illustrated in a traditional WDM network. The results show that the performance of a WBS network can be improved by using the same methods as in a WDM network. In addition, both figures show that SD-IT-WBS has a slightly higher blocking 


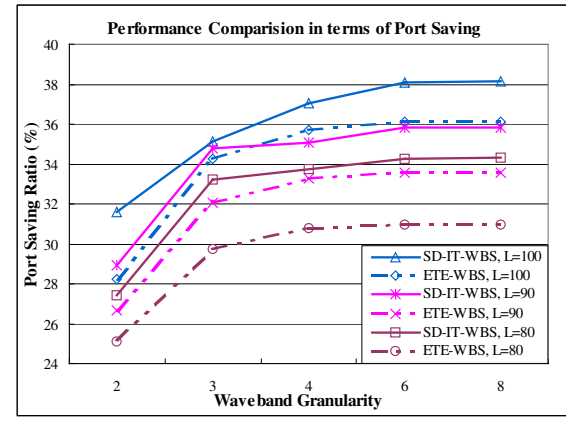

Fig. 4. Comparison of SD-IT-WBS and ETE-WBS in terms of port saving ratios on the NSF network, where $W=\{16,24,32\}$.

probability than ETE-WBS. Thus, it can be concluded that ETE-WBS slightly outperforms SD-IT-WBS in terms of network blocking probability. However, the average difference is very small. The numerical result matches the simulation result well with a slight overestimation for both WBS schemes.

Fig. 4 shows the port saving ratios for both ETE-WBS and SD-IT-WBS. The results are the average values obtained when $W$ is 16,24 , and 32 . As can be seen, the port saving ratios for both schemes increase as the network load increases and as the waveband granularity increases. The figure also implies that the port saving ratios increase as the fiber capacity increases. According to Fig. 4, SD-IT-WBS saves the used ports more than ETE-WBS does by about $2.3 \%$. As discussed at the beginning of this section, the real cost saving ratios for both WBS schemes may be higher than their port saving ratios.

While the results show that the blocking probability decreases as the fiber capacity increases and as the network load decreases, there is no linear relation between the blocking probability and the waveband granularity. Instead, the blocking probability first increases and then decreases as the waveband granularity increases. There is a peak value in the middle. Under most scenarios, the blocking probability increases as the waveband granularity increases from 2 to 6 . It then decreases as the waveband granularity becomes higher.

\section{B. Validation of the Analytical Model}

To verify the correctness of our analytical models, Fig. 5 compares the simulation results and the analytical results. As can be seen, most of the results match well. Fig. 5(a) compares the simulation results and analytical results on the NSF network when $G=2$ and $W=16 / 31$. Fig. 5(b) compares the simulation results and analytical results on the NSF network when $G=6$ and $W=16 / 31$. Both figures show that the performance differences between the analytical results and the simulation results are very small. Again, ETE-WBS slightly outperforms SD-IT-WBS. Occasionally, SD-IT-WBS has less blocking probabilities than ETE-WBS.

\section{CONCLUSION}

It is critical to analyze and compare the performance of different waveband switching schemes in designing backbone

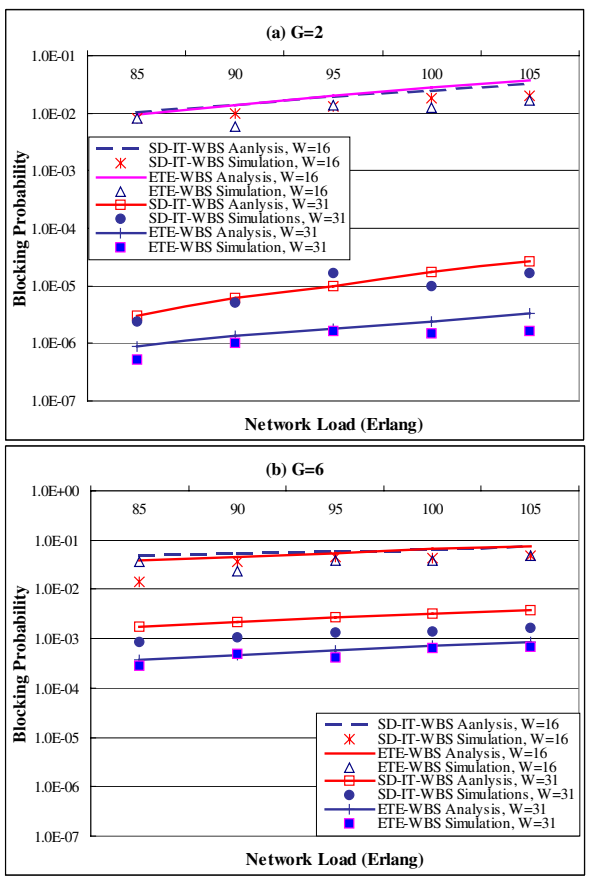

Fig. 5. Comparison of the simulation results and the analytical results for both SD-IT-WBS and ETE-WBS. (a) The NSF network, G=2, (b) The NSF network, $\mathrm{G}=6$.

WDM networks. This paper considered two waveband switching schemes, end-to-end waveband switching (ETE-WBS) and same-destination-intermediate waveband switching (SDIT-WBS). Analytical models of network blocking probability were proposed for both the schemes. An algorithm with random selection of a candidate waveband-route was proposed for SD-IT-WBS. The network blocking probability results and the cost saving results of the two schemes were presented and compared. The analytical results were compared with the simulation results, which showed good matches. The results illustrated that usually ETE-WBS slightly outperforms SDIT-WBS in network blocking probability and SD-IT-WBS outperforms ETE-WBS in cost savings.

\section{REFERENCES}

[1] Y. Suemura, I. Nishioka, Y. Maeno, S. Araki, R. Izmailov, and S. Ganguly, "Hierarchical routing in layered ring and mesh optical networks," Proc. IEEE ICC'02, vol. 5, pp. 2727-2733, Apr. 2002

[2] I. Rauf, G. Samrar, K. Viktor, and C. Aikaterini, "Non-uniform waveband hierarchy in hybrid optical networks," Proc. IEEE INFOCOM'2003, vol. 2, pp. 1344-1354, Apr. 2003.

[3] M. Li and B. Ramamurthy, "A graph model for dynamic waveband switching in WDM mesh networks," Proc. IEEE ICC'04, pp. ON08-4, 2004.

[4] L. Noirie, M. Vigoureux, and E. Dotaro, "Impact of intermediate grouping on the dimensioning of multi-granularity optical networks," Proc. of Optical Fiber Communication (OFC'01), pp. TuG3/1-3, Mar. 2001.

[5] F. P. Kelly, "Loss networks," The Annals of Appl. Prob., vol. 1, 1991.

[6] S.-P. Chung, A. Kashper, and K. W. Ross, "Computing approximate blocking probabilities for large loss networks with state-dependent routing," IEEE/ACM Trans. Networking, vol. 1, pp. 105-115, Feb. 1993.

[7] A. Birman, "Computing approximate blocking probabilities for a class of all-optical networks," IEEE J. Select. Areas Commun., vol. 14, pp. 852-857, 1996 